\title{
Study on the Application of NOMA Techniques for Heterogeneous Satellite Terminals
}

\author{
Tomás Ramírez*, Carlos Mosquera*, Nele Noels ${ }^{\dagger}$, Màrius Caus ${ }^{\ddagger}$, Joan Bas ${ }^{\ddagger}$, Luis Blanco ${ }^{\ddagger}$, Nader Alagha $^{\S}$ \\ *atlanTTic research center, University of Vigo, Galicia, Spain \\ ${ }^{\dagger}$ DIGCOM research group, TELIN Department, Ghent University, Ghent, Belgium \\ ${ }^{\ddagger}$ Centre Tecnològic de Telecomunicacions de Catalunya (CTTC/CERCA), Castelldefels, Barcelona, Spain \\ $\S$ European Space Agency, ESTEC, Noordwijk, The Netherlands
}

\begin{abstract}
This paper addresses the application of nonorthogonal multiple-access techniques (NOMA) to those satellite relayed communications for which a significant imbalance in the link quality of user terminals can be expected. The Signal-toInterference and Noise Ratio (SINR) imbalance could be caused by the coexistence of different types of terminals, possibly with different antenna sizes, and offering different classes of service. This link SINR asymmetry can be exploited to outperform orthogonal access schemes under different rate metrics, paying special attention to fairness in the service provision. Both forward and asynchronous return link are addressed, with minimum signaling information and emphasis on some relevant implementation issues such as framing and synchronization.
\end{abstract}

\section{INTRODUCTION}

In recent years Non-Orthogonal Multiple Access techniques have caught the attention of terrestrial mobile services as a way to enhance system performance. Power domain (PD) Non-Orthogonal Multiple Access (NOMA) with successive interference cancellation (SIC) at the receiver is information theoretically optimal in the sense that it maximizes the achievable rate region for single-antenna transceivers; this holds for the return link [1], as well as for the forward link [2]. In fact, the larger the difference in channel gain among users, the wider the gap between the achievable rate regions of NOMA and conventional orthogonal multiple access (OMA) schemes. The 3rd Generation Partnership Project (3GPP) started the standardization of NOMA with downlink multi-user superposition transmission (DL MUST) in Release 13 of the Long Term Evolution standard (LTE). Next, in Releases 14 and 15, NOMA has been further studied for its potential application in the 5G New Radio, including a role in the uplink. In this regard, a work item was specifically created in Release 16 for the New Radio uplink. NOMA has been proved to outperform OMA in terms of capacity and user fairness [3], at least in a first order comparison which precludes many conditioning factors which may have an effect from a global system view. At a system level, no clear gains from NOMA over Release 15 mechanisms were observed, so the work on NOMA was discontinued for $5 \mathrm{G}$, with possible use beyond $5 \mathrm{G}$.

Taking into account that PD-NOMA is specially suited to exploit a power imbalance among different terminals, in this work we focus on a satellite system scenario where a heterogeneous population of terminals with a large imbalance in the link quality co-exist. This is the case, for example, when satellite resources are shared between large antenna fixed ground terminals and small antenna mobile platforms such as aircrafts. For simplicity, we assume a scenario with singleantenna user terminals and only one satellite beam. The latter epitomizes a single-beam coverage or a multi-beam setting with at least four colors, so that co-channel interference can be neglected.

After an initial description of NOMA in Section II, Section III presents a forward link scenario with heterogeneous receivers, together with system level simulations to address the potential gains with PD-NOMA using modulation and coding (MODCOD) schemes from the DVB-S2X standard for Digital Video Broadcasting via Satellite [4]. In forward link NOMA, groups of users are served using the same resource blocks by transmitting a weighted sum of the respective user signals. The key challenges addressed in Section III are the grouping of users and the design of the weight factor for each user within a group. In order to accomodate NOMA for satellite, we propose some adjustments to the DVB-S2X super-frame (SF) profile; this is necessary because forward link SIC requires that users can acquire information on the MODCODs and the Signalto-Interference and Noise Ratios (SINR) of all superimposed signals.

In Section IV and V, the use of return link NOMA is considered for satellite terminals with a large imbalance in their respective Equivalent Isotropically Radiated Power (EIRP). We distinguish between a system with truly asynchronous (unslotted) random access (RA) in Section IV and a more conventional system with demand-assigned multiple access (DAMA) in Section V.

Section IV investigates the coexistence of two classes of RA Enhanced Spread Spectrum ALOHA (E-SSA) based Internetof-Things (IoT) terminals [5], [6]. The theoretical throughput of an integrated system, in which the two classes of E-SSA terminals employ the same resources and with SIC detection at the gateway, is evaluated and compared to that of a system that orthogonally allocates a particular part of the resources to each terminal class.

The starting point of Section $\mathrm{V}$ is the DVB-RCS/DVBRCS2 standard for the Digital Video Broadcasting - Return Channel via Satellite, where the multiple access is based on Multi-Frequency Time-Division Multiple Access (MF-TDMA) [7], [8]. Considering the expected gain coming from the use of 
NOMA and SIC when the power imbalance among NOMA terminals is large, we study some particular implementation details associated to the return link NOMA paradigm. Special attention goes to the design of the parameter estimation and synchronization structures that are required for the deployment of a coherent SIC detector.

\section{SYSTEM CONSIDERATIONS}

With two-user PD-NOMA, the received signal at a given terminal experiencing a given complex channel gain $h$ at a given time instant, in the forward link of a single beam satellite, can be expressed as

$$
y=h \cdot \sqrt{P}\left(\sqrt{\lambda} \cdot x_{1}+\sqrt{1-\lambda} \cdot x_{2}\right)+n, 0 \leq \lambda \leq 1
$$

where $P$ is the transmit power, $n$ is the receiver noise, with a power which depends on the type of terminal, and $x_{1}$ and $x_{2}$ are the superimposed unit-energy symbols for users 1 and 2, respectively. Under this basic single-antenna model, fractions $\lambda$ and $(1-\lambda)$ of the transmit power are allocated to the the first and second) users respectively. A perfect time alignment is easily ensured between both signals, considering single satellite beam operation.

The corresponding application of PD-NOMA to the return link is expressed by the received signal at the gateway as

$$
y=h_{1} \sqrt{P}_{1} \cdot x_{1}+h_{2} \sqrt{P}_{2} \cdot x_{2}+z
$$

with $h_{i}, i=1,2$, the channel from user $i$ to the gateway, $P_{i}$ the corresponding transmit power, $z$ the gateway noise, and $x_{1}$ and $x_{2}$ the superimposed symbols independently transmitted by the two users; as a result, perfect time alignment can no longer be ensured. Note that the forward link channel has a constraint on the sum of the transmit power due to the satellite power amplifier characteristics. On the return link this constraint is applied individually to each transmitter. On the other hand, there is an input saturation power constraint on board of the satellite at the first stages of the receiver front-end that limits the total power density. Other than that, implementation challenges are similar in both directions when it comes to the receive functions, especially in terms of SINR acquisition, framing or the cancellation interference. The detection, decoding and removal of one of the received signals before demodulating the other is known as SIC, and it is widely considered as one of the key ingredients for the success of non-orthogonal schemes. In both forward and return link perfect SIC and parameter estimation will be assumed for performance evaluation purposes. Nevertheless, the potential degradation due to imperfect parameter estimation will be covered in Section V. The channel will follow an Additive White Gaussian Noise (AWGN) model, although the impact of non-linearities will be also touched upon.

\section{FORWARD LINK}

We address a forward link NOMA satellite system scenario where superimposed signals are transmitted to simultaneously serve two classes of receivers: strong receivers (i.e., with a high antenna gain versus noise temperature ratio, G/T) and weak receivers. Receivers of different classes have different front-ends ${ }^{1}$, which give rise to an SINR imbalance between them. SIC is performed only at the strong receivers.

As a first step, the accommodation of PD-NOMA with SIC in the current DVB-S2X standard is analyzed. The frame must inform about the MODCODs of the two superimposed signals, and facilitate the estimation of the individual SINRs of both user signals at the receivers: a new super-frame profile is presented to address these NOMA demands. Once the support of PD-NOMA transmissions is guaranteed, the focus is shifted to obtain an initial measure of the performance of PD-NOMA with standard DVB-S2X MODCODs based on system level simulations.

\section{A. PD-NOMA in the DVB-S2X Standard}

We propose the use of the DVB-S2X SF for the PDNOMA implementation since it offers the required flexibility to accommodate the framing features for the superposition of signals without requiring changes on the standard. The proposed SF profile is presented in Fig. 1. It consists of the conventional Start-Of-SF (SOSF) and SF-Format-Indicator (SFFI) fields, followed by different NOMA-PLFRAMES, each one consisting of a new header and a payload for the NOMA operation. The NOMA-PLFRAME header contains the concatenation of two conventional Physical Layer Signaling Codes (PLSCODE), each specifying the MODCOD of one of the two superimposed PLFRAMES. Note that we choose not to define new MODCODs for PD-NOMA. This has the advantage that the PLSCODE table does not have to change, making the implementation transparent for receivers without SIC. The generation of the NOMA-PLFRAME payload is depicted in Fig. 2; the symbols of the compounding DVBS2X XFECFRAMEs (complex symbol frames) are aligned and summed after allocating a fraction of the total transmit power ${ }^{2}$.

As indicated in Fig. 1, we propose an interweaved dedicated pilot block within each NOMA PLFRAME payload. Besides the possible use of the pilots for estimation of the timing, carrier synchronization and channel estimation, a given number of pilots may be employed for another required feature for PD-NOMA operation, that is, the SINR estimation of the superimposed signals. With the assumption of a data aided (DA) algorithm such as SNORE [9], the estimation of the SINR can be achieved with a signalling sequence $c_{k}$ that is also a superposition of two different orthogonal sequences $c_{k}^{1}$ and $c_{k}^{2}$ with unit power:

$$
c_{k}=\sqrt{\lambda} \cdot c_{k}^{1}+\sqrt{1-\lambda} \cdot c_{k}^{2},
$$

where $0 \leq \lambda \leq 1$ is the power allocation ratio of the superimposed signals. Neglecting any residual synchronization errors at reception, the pilot signal at a given receiver can be expressed as

$$
y_{k}=\sqrt{P} \cdot h \cdot c_{k}+n_{k}
$$

${ }^{1}$ Different antenna sizes, or amplifiers with different noise figures, for example.

${ }^{2}$ The superposition of strong and weak frames with possibly different modulations imposes some constraints on the combinations that can be accommodated, due to the different PLFRAME durations. 


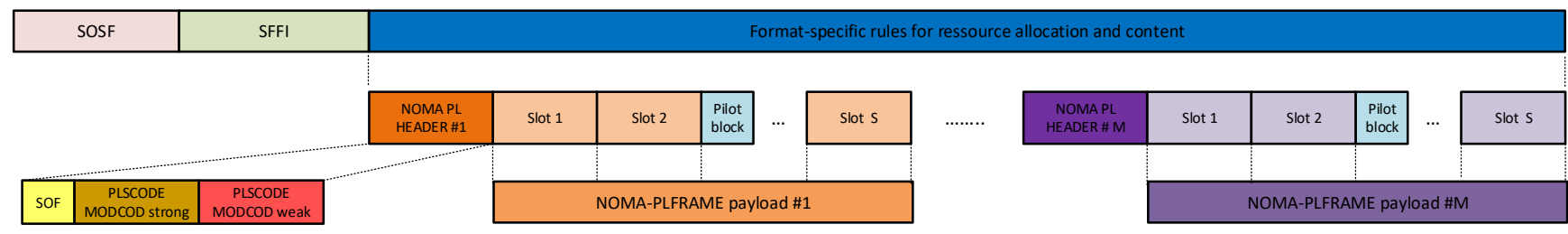

Fig. 1. Super-frame profile for PD-NOMA operation in DVB-S2X.

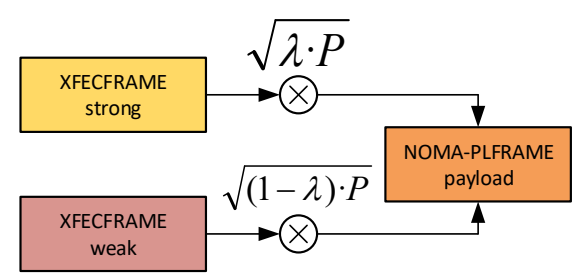

Fig. 2. NOMA-PLFRAME payload generation at the transmitter as the superposition in power of two conventional DVB-S2X XFECFRAMEs.

where the additive noise has a power density $N_{0}$. Let us consider a strong receiver. In order to carry out the SIC procedure, this receiver needs to estimate the received power and the SINR of both compound signals. If we denote $\rho_{S}=\lambda \cdot \mathrm{SNR}_{S}$ and $\rho_{W}=(1-\lambda) \cdot \mathrm{SNR}_{S} /\left(1+\lambda \cdot \mathrm{SNR}_{S}\right)$ as the NOMA SINR of the strong and weak messages, respectively, at the strong receiver, the corresponding estimates $\hat{\rho}_{S}$ and $\hat{\rho}_{W}$ with SNORE can be obtained as

$$
\begin{array}{rlrl}
\hat{\rho}_{W} & =\frac{P_{W}}{P_{T}-P_{W}} & \hat{\rho}_{S}=\frac{P_{S}}{P_{T}-P_{S}-P_{W}} \\
P_{S} & =\frac{1}{L} \sum_{k=1}^{L}\left|\left(c_{k}^{1}\right)^{*} \cdot y_{k}\right|^{2} & P_{W}=\frac{1}{L} \sum_{k=1}^{L}\left|\left(c_{k}^{2}\right)^{*} \cdot y_{k}\right|^{2} \\
P_{T}=\frac{1}{L} \sum_{k=1}^{L}\left|y_{k}^{S}\right|^{2} & \mathrm{SNR}_{S}=\frac{\lambda \cdot|h|^{2} \cdot P}{N_{0}}
\end{array}
$$

where $L$ is the length of the superimposed pilot sequence, and $P_{T}, P_{S}$ and $P_{W}$ are the estimates of the total received power, the received power of the strong signal and the received power of the weak signal, respectively. A similar procedure can be applied at the weak receiver, which needs to estimate only the received power and the SINR of the weak user signal.

\section{B. Scenario Description}

Next we consider user pairing and the power allocation within each pair in a single beam scenario. As optimization criterion we choose to maximize the system sum-rate, subject to a Quality of Service (QoS) minimum rate requirement for the weak user, at least equal to that achieved with OMA if the resources are employed to serve the weak user during a fraction $\alpha$ of the time, with $\alpha$ a design parameter. The number of weak and strong receivers within the beam footprint will be identical. The long frame duration in DVB-S2/S2X makes it common to serve several users with the same multicast frame; in consequence, we will create $N$ equal size multicast groups of each receiver class. Since the multicast transmission rate is limited by the user with the lowest SNR in the multicast group, the clustering of users should be such that the SNR differences within each group are as small as possible.

Let $u_{i j} \in\{0,1\}$ label the pairing between $i$-th and $j$-th groups, so that groups $i$ and $j$ are paired if $u_{i j}=u_{j i}=1$. After the user grouping, let $\mathrm{SNR}_{S}^{i}>\mathrm{SNR}_{W}^{j}$ denote the SNR values of the weakest user within the $i$-th and the $j$-th groups, respectively. The optimization problem can be expressed as

$$
\begin{aligned}
\max _{u_{i j}, \lambda_{i j}} & \sum_{i=1}^{N} \sum_{j=1}^{N} u_{i j}\left(R_{S}^{i}+R_{W}^{j}\right) \\
\text { s.t. } & u_{i j} \in\{0,1\}, 0 \leq \lambda_{i j} \leq 1 ; i, j=1,2, \ldots, N \\
& \sum_{i=1}^{N} u_{i j}=1, \forall i, \sum_{j=1}^{N} u_{i j}=1, \forall j \\
& R_{W}^{j} \geq B \cdot \alpha \cdot \Pi\left(\mathrm{SNR}_{W}^{j}\right) \\
& R_{S}^{i}=B \cdot \Pi\left(\lambda_{i j} \mathrm{SNR}_{S}^{i}\right) \\
& R_{W}^{j}=B \cdot \Pi\left(\frac{\left(1-\lambda_{i j}\right) \mathrm{SNR}_{W}^{j}}{1+\lambda_{i j} \mathrm{SNR}_{W}^{j}}\right)
\end{aligned}
$$

where $\Pi$ is a function that maps the input NOMA SINR value to a DVB-S2X MODCOD spectral efficiency, and $B$ is the available bandwidth. This is a matching problem with can be solved with the Hungarian algorithm [10], which serves to optimize the sum-rate based on the assignment matrix $C$, with the $i j$-th element given by

$$
c_{i j}=-R_{S}^{i}+R_{W}^{j} .
$$

Therefore, the optimization problem is decoupled into two steps: (i) matrix $\boldsymbol{C}$ is built for all possible pairs based on the optimal rates and parameters $\lambda_{i j}$ obtained from the weak users minimum rate constraint; (ii) the Hungarian algorithm is used to select the optimum set of pairs.

\section{Numerical Results}

Numerical results are obtained after performing 1,200 Monte-Carlo simulations with the system level assumptions from Table I. Different antenna gain gap values between strong and weak users are tested in the range $(3,13) \mathrm{dB}$, thus simulating different power imbalance conditions. In the AWGN case, we have successfully tested the DVB-S2X MODCOD 
TABLE I

SYSTEM PARAMETERS FOR THE FORWARD LINK.

\begin{tabular}{cc}
\hline Diagram pattern & Provided by ESA \\
\hline Frequency band [GHz] & 20 \\
\hline EIRP/beam & $62 \mathrm{dBW}$ \\
\hline Fading & Atmospheric losses \\
\hline Number of user per beam & 45 (each kind) \\
\hline Number of groups per class & 15 (each kind) \\
\hline Common Receiver Parameters \\
\hline Receiver cloud noise temperature & $280^{\circ} \mathrm{K}$ \\
\hline Receiver terminal noise temperature & $310^{\circ} \mathrm{K}$ \\
\hline Receiver ground noise temperature & $45^{\circ} \mathrm{K}$ \\
\hline LNB Noise Figure & $2 \mathrm{~dB}$ \\
\hline Interference cancellation & Ideal cancellation \\
\hline Strong Receiver Parameters \\
\hline Receiver antenna efficiency \\
\hline Receiver antenna diameter & 0.65 \\
\hline
\end{tabular}

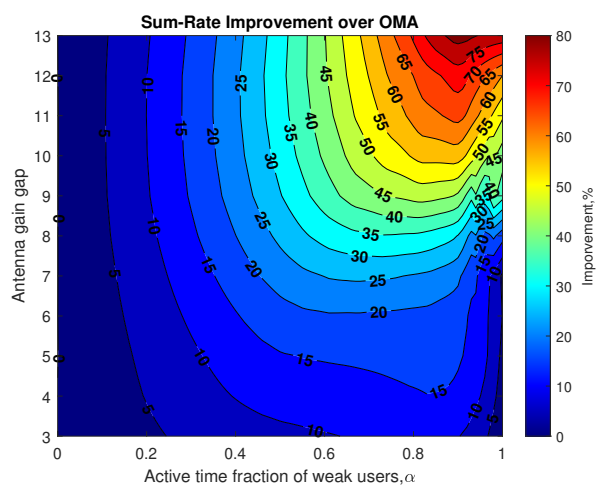

Fig. 3. Average sum-rate improvement of PD-NOMA with DVB-S2X MODCODs for an active weak user time $\alpha$.

thresholds after extensive physical layer simulations, so they were employed to drive the mapping $\Pi$ between the NOMA SINR values and the DVB-S2X MODCODs. Not all DVBS2X MODCODs are employed: VL SNR and 8PSK/8APSK MODCODs are excluded due to XFECFRAME sizes and the extra challenge to accommodate them in the proposed SF.

The resulting performance is presented in Fig. 3 for different values of the antenna gain gap and the weak user active time fraction $\alpha$. As a general conclusion, PD-NOMA requires that the weak users have a significant QoS demand, measured as medium to high values of active time in the OMA case. Otherwise, the potential gains with PD-NOMA cannot be enough to justify PD-NOMA as an interesting alternative regardless of the antenna gain gap between receiver classes. Finally, we need to remind that a linear channel has been considered; non-linear elements are expected to create a larger impairment for NOMA due to the higher Peak-to-Average Power Ratio (PAPR), so that the gains would be somewhat reduced. Initial tests prove that the degradation is larger for lower SNR regimes, for which the PAPR of the OMA signals associated to more robust constellations is lower.

\section{RETURN LINK}

We consider a satellite return link that is devoted to provision IoT services. Accordingly, there is a large population of users that are allowed to transmit short packets sporadically without any synchronization. One of the appealing RA schemes that suits the needs of satellite IoT systems is ESSA [5]. E-SSA stands out because collisions are resolved through the use of spreading codes and by implementing iterative packet-based SIC. It should be noted that, unlike forward link SIC, return link SIC deals with power imbalance by ranking the contributions in descending order of received power and starting the detection from the most powerful one. An analytical framework for investigating the performance of E-SSA was outlined in [6]. The model in [6] assumed a homogeneous population of terminals. All terminals have the same antenna gain and transmit power, and employ the same MODCOD. $^{3}$

The purpose of the current section is to investigate the throughput of an E-SSA system that provisions IoT services to two types of terminals, strong and weak, with the former having larger antennas and transmit powers, i.e., $G_{T}^{S}>G_{T}^{W}$ and $P_{S}>P_{W}$. Further, all terminals (both weak and strong) are assumed to use the same code rate $r$, the same symbol alphabet size $M$ and the same chip rate $R_{c}$. However, in order to increase their robustness to noise and interference from strong terminals, the weak terminals are allowed to use a larger spreading factor than the strong terminals $\left(S F_{S}<S F_{W}\right)$. The energy-bit-to-noise ratio $\left(E_{b} / N_{0}\right)$ for strong and weak terminals at the center of a satellite beam can be computed as

$\left[E_{b} / N_{0}\right]_{\mathrm{REF}, \mathrm{u}}=\frac{G_{R}^{0} G_{T}^{\mathrm{u}} P_{\mathrm{u}}}{L_{0}} \frac{S F_{\mathrm{u}}}{r \log _{2} M} \frac{1}{R_{c} K T_{s y s}}, \mathrm{u} \in\{S, W\}$,

where $L_{0}$ and $G_{R}^{0}$ are the loss factor and the satellite antenna gain in the beam center, $K$ is the Bolzmann constant and $T_{\text {sys }}$ is the system temperature. Taking into account the different user positions within a beam, the actual $E_{b} / N_{0}$ is modelled as $\Delta \cdot\left[E_{b} / N_{0}\right]_{\mathrm{REF}, \mathrm{u}}$, with $\Delta$ a discrete random variable that takes the values $\left\{\delta^{j}\right\}$ for $j \geq 1$, according to a probability mass function $(\mathrm{PMF}) P_{\Delta}(j)=\operatorname{Pr}\left[\Delta=\delta^{j}\right]$. Following the approach proposed in [6], we employ a fixed logarithmic step size of 0.1 $\mathrm{dB}$ to define the domain of $\Delta$, i.e., $10 \log _{10}\left(\delta^{j+1} / \delta^{j}\right)=0.1$.

In this work, the $P_{\Delta}(j)$ will be derived from $N_{p}$ discrete channel gain observations $g_{n}, n=0,1, \ldots, N_{p}-1$, with

$$
g_{n}=\frac{G_{R}^{n}}{L_{n}}=\frac{G_{R}^{n}}{A\left|4 \pi d_{n} / \lambda\right|^{2}}
$$

Here, the term $G_{R}^{n}$ refers to the satellite antenna gain in the direction of the $n$-th user position. As for the free loss factor $L_{n}, \lambda$ is the carrier wavelength, $A$ denotes the atmospheric loss and $d_{n}$ is the distance from the satellite to the $n$-th user position. The beam center is associated to the 0 -th user position. We have used a multibeam satellite antenna radiation pattern provided by ESA with a 4-color frequency reuse scheme. Fig. 4(a) shows the corresponding normalized channel

${ }^{3}$ In this case, the MODCOD includes the specification of the spreading factor, i.e., the number of chips that is used to spread a given symbol. 


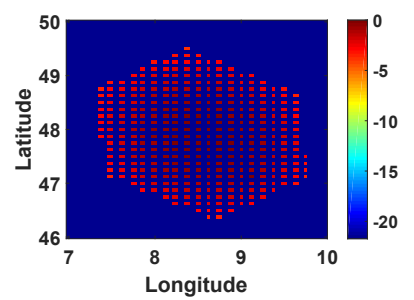

(a) Normalized channel gain

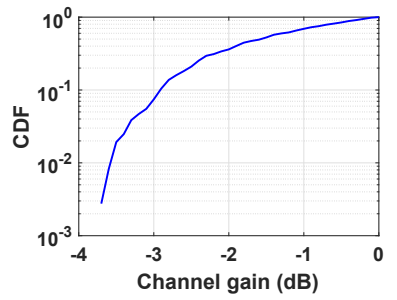

(b) $\mathrm{CDF}$
Fig. 4. Channel model.

gain $g_{n} / g_{0}$. There are approximately $N_{p}=360$ positions with different gains. From the observations $\left\{g_{n}\right\}$, we compute

$$
P_{\Delta}(j)=\int_{\delta^{(j-1)}}^{\delta^{j}} \frac{1}{N_{p}} \sum_{n=0}^{N_{p}-1} \delta\left(g-\frac{g_{n}}{g_{0}}\right) d g, \quad j \geq 1,
$$

where $\delta\left(g-\frac{g_{n}}{g_{0}}\right)$ is 1 if $g=\frac{g_{n}}{g_{0}}$ and 0 otherwise. The $E_{b} / N_{0}$ of each packet is mapped to a bin $\left[\delta^{(j-1)}, \delta^{j}\right]$, with $j \geq 1$, and the $j$-th bin is associated to the value $\delta^{j}$. To complete the statistics we have represented in Fig. 4(b) the cumulative distribution function (CDF) corresponding to the resulting $P_{\Delta}(j)$.

\section{A. Analytical Model}

In the following, we extend the results from [6] to the above mentioned heterogeneous scenario. As for the decoding strategy, SIC is applied to detect the strong user packets from highest to lowest $E_{b} / N_{0}$ bin, followed by the weak user packets also from highest to lowest $E_{b} / N_{0}$ bin; the whole procedure is iterated $I$ times. Following the steps from [6], the aggregate throughput in the coexistence scenario can be computed as

$$
\begin{aligned}
& \eta_{T}\left(\lambda_{\text {MAC }}^{S}, \lambda_{\text {MAC }}^{W}\right)=\sum_{\mathrm{u} \in\{S, W\}} \eta_{\mathrm{u}}\left(\lambda_{\text {MAC }}^{S}, \lambda_{\text {MAC }}^{W}\right) \\
& =\sum_{\mathrm{u} \in\{S, W\}} \lambda_{\text {MAC }}^{\mathrm{u}}\left(1-\operatorname{PLR}_{\mathrm{u}}^{I}\left(\lambda_{\text {MAC }}^{S}, \lambda_{\text {MAC }}^{W}\right)\right),
\end{aligned}
$$

where $\eta_{\mathrm{u}}\left(\lambda_{\text {MAC }}^{S}, \lambda_{\text {MAC }}^{W}\right)$ and $\operatorname{PLR}_{\mathrm{u}}^{I}\left(\lambda_{\text {MAC }}^{S}, \lambda_{\text {MAC }}^{W}\right)$ respectively denote the throughput and the packet loss ratio (PLR) achieved by terminals of class-u after $I$ iterations, given a load pair of $\left(\lambda_{\mathrm{MAC}}^{S}, \lambda_{\mathrm{MAC}}^{W}\right)$, with $\lambda_{\mathrm{MAC}}^{u}$ denoting the load of class $\mathrm{u}$ packets. The load of class-u packets can be expressed in bits/chip as $\lambda_{\mathrm{MAC}}^{\mathrm{u}}=\frac{\lambda}{G_{p}^{\mathrm{u}}}$, where $G_{p}^{\mathrm{u}}=\frac{S F_{\mathrm{u}}}{r \times \log _{2}(M)}$ corresponds to the processing gain and $\lambda_{\mathrm{u}}$ denotes the average number of class $\mathrm{u}$ packet arrivals during one class $\mathrm{u}$ packet duration $T_{\mathrm{u}}$, for $\mathrm{u} \in\{S, W\}$. Class- $S$ and class- $W$ packet arrivals are assumed to follow independent Poisson distributions. The probability that $k$ class $\mathrm{u}$ packets arrive within $T_{\mathrm{u}}$ is given by $P_{p}\left(k ; \lambda_{\mathrm{u}}\right)=\frac{\lambda_{\mathrm{u}}^{k} e^{-k}}{k !}, \mathrm{u} \in\{S, W\}$.

Assuming that the payload size is fixed and equal for both terminal classes, we have $T_{W}=N T_{S}$, with $N=$ $S F_{W} / S F_{S} \geq 1$ [11]. To benefit from the model derived in [6], we consider all class-u packet arrivals over a window of size $2 T_{W}$ centered around the start of a class-u' $\mathrm{u}^{\prime}$ packet of interest, and we compute: (1) the average number of such arrivals $\left(\lambda_{t}^{\mathrm{u}}\right),(2)$ the average overlapping between the arriving packets and the packet of interest $\left(\beta_{\mathrm{u}^{\prime} \mathrm{u}}\right)$. It can be verified that $\lambda_{t}^{S}=2 N \lambda_{S}, \lambda_{t}^{W}=2 \lambda_{W}, \beta_{S S}=\frac{1}{2 N}, \beta_{S W}=0.5$, $\beta_{W S}=\frac{1}{2 N}$ and $\beta_{W W}=0.5$.

The procedure for obtaining the PLR of the strong and the weak terminals is very similar. For the sake of brevity, we only present here the equations for the PLR of the strong terminals. For $1 \leq i \leq I$, this PLR is computed as the average of the probability $P_{e}^{S}\left(j, \lambda_{\text {MAC }}^{S}, \lambda_{\text {MAC }}^{W}, i\right)$ of erroneously detecting a class- $S$ packet in the $i$ th iteration when its $E_{b} / N_{0}$ is in bin $j$, over the PMF of $j$ from (9):

$$
\operatorname{PLR}_{S}^{i}\left(\lambda_{\mathrm{MAC}}^{S}, \lambda_{\mathrm{MAC}}^{W}\right)=\sum_{j} P_{\Delta}(j) P_{e}^{S}\left(j, \lambda_{\mathrm{MAC}}^{S}, \lambda_{\mathrm{MAC}}^{W}, i\right) .
$$

It can be inferred from [6] that computing $P_{e}^{S}\left(j, \lambda_{\text {MAC }}^{S}, \lambda_{\text {MAC }}^{W}, i\right)$ boils down to computing the average power spectral density of $k_{S}$ class- $S$ and $k_{W}$ class- $W$ packets arriving between $T_{W}$ before and $T_{W}$ after the desired packet; we have

$$
\begin{gathered}
P_{e}^{S}\left(j, \lambda_{\mathrm{MAC}}^{S}, \lambda_{\mathrm{MAC}}^{W}, i\right)=\sum_{k_{S}, k_{W}=0}^{\infty} P_{p}\left(k_{S} ; \lambda_{t}^{S}\right) \\
\cdot P_{p}\left(k_{W} ; \lambda_{t}^{W}\right) \Gamma\left\{\frac{\delta^{j}\left[E_{b} / N_{0}\right]_{\mathrm{REF}, S}}{1+I_{S}\left(k_{S}, j, i\right) / N_{0}+I_{W}\left(k_{W}, i\right) / N_{0}}\right\},
\end{gathered}
$$

where $\Gamma\{\rho\}$ is a function that maps energy-bit-to-interferenceplus-noise ratio values into frame error rate (FER) values,

$$
\begin{aligned}
\frac{I_{S}\left(k_{S}, j, i\right)}{N_{0}}= & \sum_{l} \frac{\delta^{l}\left[E_{b} / N_{0}\right]_{\mathrm{REF}, S}}{G_{p}^{S}} \cdot N_{S}\left(l, k_{S}, j, i\right), \\
\frac{I_{W}\left(k_{W}, i\right)}{N_{0}}= & \sum_{l} \frac{\delta^{l}\left[E_{b} / N_{0}\right]_{\mathrm{REF}, W}}{G_{p}^{W}} \\
& \cdot \beta_{S W} k_{W} P_{\Delta}(l) P_{e}^{W}\left(l, \lambda_{\mathrm{MAC}}^{S}, \lambda_{\mathrm{MAC}}^{W}, i-1\right),
\end{aligned}
$$

with $N_{S}\left(l, k_{S}, j, i\right)$ denoting the residual equivalent number of class- $S$ packets colliding with the considered class- $S$ packet at iteration $i$ in the $j$ th $E_{b} / N_{0}$ bin when the total number of class- $S$ packets arriving in the considered window of duration $2 T_{W}$ is $k_{S}$. The quantity $N_{S}\left(l, k_{S}, j, i\right)$ can be expressed as

$$
\begin{aligned}
& N_{S}\left(l, k_{S}, j, i\right)= \\
& \quad\left\{\begin{array}{cc}
\beta_{S S} k_{S} P_{\Delta}(l) P_{e}^{S}\left(l, \lambda_{\mathrm{MAC}}^{S}, \lambda_{\mathrm{MAC}}^{W}, i-1\right) & , l \leq j \\
\beta_{S S} k_{S} P_{\Delta}(l) P_{e}^{S}\left(l, \lambda_{\mathrm{MAC}}^{S}, \lambda_{\mathrm{MAC}}^{W}, i\right) & , l>j .
\end{array}\right.
\end{aligned}
$$

Equations (11)-(14) (and similar equations for the error probability of class- $W$ packets) allow to compute $P_{e}^{u}\left(j, \lambda_{\mathrm{MAC}}^{S}, \lambda_{\mathrm{MAC}}^{W}, i\right)$ for all $i=1,2, \ldots, I$, all $u \in\{S, W\}$ and all $j \geq 1$ in a recursive way. To start, $i$ is set to zero and $P_{e}^{S}\left(j, \lambda_{\mathrm{MAC}}^{S}, \lambda_{\mathrm{MAC}}^{W}, 0\right)$ and $P_{e}^{W}\left(j, \lambda_{\mathrm{MAC}}^{S}, \lambda_{\mathrm{MAC}}^{W}, 0\right)$ are set to 1 for all $j$. Subsequently, the following procedure is iterated $I$ times. (1) Increment the value of $i$ by 1. (2) Compute

\footnotetext{
${ }^{4}$ This is the smallest window that is large enough to include all possible
} colliding packets, involving any class of terminals. 


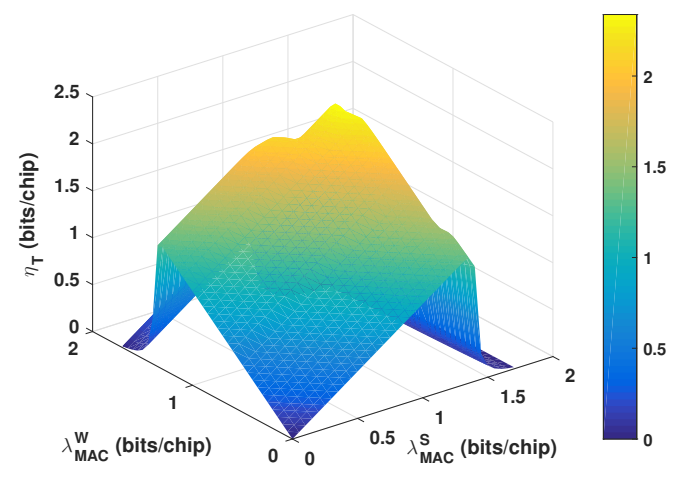

Fig. 5. E-SSA aggregate throughput at iteration 2 versus MAC load.

$P_{e}^{S}\left(j, \lambda_{\mathrm{MAC}}^{S}, \lambda_{\mathrm{MAC}}^{W}, i\right)$ from the highest to the lowest value of $j$. (3) Compute $P_{e}^{W}\left(j, \lambda_{\text {MAC }}^{S}, \lambda_{\text {MAC }}^{W}, i\right)$ from the highest to the lowest value of $j$.

\section{B. Numerical Results}

Hereafter, we provide some numerical results. In the proposed scenario we analyze the return link of a GEO satellite, with $G_{R}^{0} / T_{\text {sys }}=18 \mathrm{~dB} / \mathrm{K}, A=1 \mathrm{~dB}, G_{T}^{S}=43 \mathrm{dBi}$, $G_{T}^{W}=26 \mathrm{dBi}, P_{S}=27 \mathrm{dBm}, P_{W}=26 \mathrm{dBm}, S F_{S}=32$ and $S F_{W}=64$. Note that there is an imbalance of $18 \mathrm{~dB}$. For the channel, we have adopted the model represented in Fig. 4. Both systems employ the 3GPP turbo code of rate $r=1 / 3$ and Binary Phase Shift Keying (BPSK) modulation $(M=2)$, such that the closed-form expression of the function $\Gamma\{$.$\} from [6]$ can be employed.

The aggregate throughput formulated in (10) is represented in Fig. 5. If we impose a target PLR of $0.1 \%$ for both terminal classes, the maximum achievable throughput found is 2.1 bits/chip upon performing $I=2$ iterations. As a benchmark we have considered a system where different classes of terminals operate on different frequency bands. When the bandwidth is equally split, the aggregate throughput can be formulated as $\eta_{T}\left(\lambda_{\text {MAC }}^{S}, \lambda_{\text {MAC }}^{W}\right)=\frac{1}{2}\left(\eta_{S}\left(\lambda_{\text {MAC }}^{S}, 0\right)+\eta_{W}\left(0, \lambda_{\text {MAC }}^{W}\right)\right)$. If the desired link reliability is PLR $=0.1 \%$, the channel can be loaded up to $1.3 \mathrm{bits} / \mathrm{chip}$. Hence, in the non-orthogonal coexistence scenario, gains up to $61 \%$ can be provided with respect to the case where the frequency is segregated among the two services. If we repeat the test for $G_{T}^{W}=36 \mathrm{~dB}$, then it follows that the gain is reduced to $42 \%$. This corroborates that the advantage increases as the imbalance becomes larger.

\section{Parameter Estimation Performance Analysis}

The previous system level analysis for both forward and return links was performed at the system level, with the corresponding abstraction of the physical layer which, among other things, assumed the perfect estimation of all involved parameters. In the following we examine the estimation performance of time delays, frequency offsets, phases and amplitudes for two superimposed waveforms in a demand-assigned pairwise
TABLE II

Physical Layer Parameters For the DAMA Return LinK

\begin{tabular}{cc}
\hline Nb. users per traffic slot & 2 \\
\hline Nb. symbols per burst $(K)$ & 536 or 1616 \\
\hline pulse shaping & cosine rolloff \\
\hline modulation & 4-QAM or 16-QAM \\
\hline channel, noise PSD & AWGN, $N_{0}$ \\
\hline symbol period & $T$ \\
\hline$S N R_{S}$ & 10 to $15 \mathrm{~dB}$ \\
\hline$S N R_{W}$ & 0 to $5 \mathrm{~dB}$ \\
\hline$S N R_{S} / S N R_{W}$ & 0.05 or 0.2 \\
\hline rolloff factor $\left(\alpha_{S}, \alpha_{W}\right)$ & i.i.d., uniform in $\left[-\frac{T}{2}, \frac{T}{2}\right]$ \\
\hline time delay $\left(\tau_{S}, \tau_{W}\right)$ & i.i.d., uniform in $\left[-\frac{3}{100 T}, \frac{3}{100 T}\right]$ \\
\hline frequency offset $\left(F_{S}, F_{W}\right)$ & i.i.d., uniform in $[-\pi, \pi]$ \\
\hline phase $\left(\theta_{S}, \theta_{W}\right)$ & fixed, $A_{u}=\sqrt{S N R_{u} \cdot N_{0}}, u \in\{S, W\}$ \\
\hline amplitude $\left(A_{S}, A_{W}\right)$
\end{tabular}

PD-NOMA satellite return link scenario, when a significant imbalance in the links quality exists.

\section{A. Observation Model}

Each traffic slot is simultaneously allocated to a strong and a weak terminal. The received signal is

$$
y(t)=\sum_{\mathrm{u} \in\{W, S\}} f_{\mathrm{u}}\left(t ; \boldsymbol{x}_{\mathrm{u}}, \tau_{\mathrm{u}}, F_{\mathrm{u}}, \theta_{\mathrm{u}}, A_{\mathrm{u}}\right)+n(t),
$$

with $n(t)$ complex-valued AWGN with power spectral density $N_{0}$ and $f_{\mathrm{u}}$ the waveform received from the weak $(\mathrm{u}=W)$ or the strong $(\mathrm{u}=S)$ terminal, i.e.,

$$
\begin{aligned}
& f_{\mathrm{u}}\left(t ; \boldsymbol{x}_{\mathrm{u}}, \tau_{\mathrm{u}}, F_{\mathrm{u}}, \theta_{\mathrm{u}}, A_{\mathrm{u}}=\right. \\
& A_{\mathrm{u}} e^{j\left(2 \pi F_{\mathrm{u}} t+\theta_{\mathrm{u}}\right)} \sum_{k=0}^{K-1} x_{\mathrm{u}, k} h_{u}\left(t-\tau_{\mathrm{u}}-k T\right) .
\end{aligned}
$$

The interfering waveforms $\left(f_{S}, f_{W}\right)$ are linear modulated square-root cosine roll-off pulse trains with the same length $K$ and symbol period $T$, but with different rolloff factor $\left(\alpha_{S}, \alpha_{W}\right)$, symbol sequence $\left(\boldsymbol{x}_{S}, \boldsymbol{x}_{W}\right)$, time delay $\left(\tau_{S}, \tau_{W}\right)$, frequency offset $\left(F_{S}, F_{W}\right)$, phase $\left(\theta_{S}, \theta_{W}\right)$ and amplitude $\left(A_{S}, A_{W}\right)$. The SNR of the strong terminal is assumed to be much higher than that of the weak terminal, i.e., $S N R_{S} \gg$ $S N R_{W}$, with $S N R_{u}=\frac{A_{u}^{2}}{N_{0}}$, for $u \in\{S, W\}$. More information on the physical layer assumptions can be found in Table II. The starting point for the reported values are the DVB-RCS recommendations [7], [8]. Estimates of $\tau_{u}, F_{u}, \theta_{u}$ and $A_{u}$ will be denoted with a hat. The receiver is assumed to perform SIC; the signal from the strong terminal is detected and cancelled prior to the detection of the signal of the weak terminal. Next, the effect of estimation errors on SIC is discussed, with a closer look at time delay estimation.

\section{B. General Assessment}

Table III summarizes the main points of our analysis:

line 1-4: For all considered SNR pairs from Table II, $\mathrm{SNR}_{S}$ is high and $\mathrm{SNR}_{W}$ is low. As explained below, this has direct consequences for the NOMA SINR values. 
TABLE III

IMPACT OF ESTIMATION ERRORS ON PERFORMANCE: INITIAL ANALYSIS

\begin{tabular}{l|l|l}
\hline $\mathrm{SNR}_{S}$ & High & \\
\hline $\mathrm{SNR}_{W}$ & Low & \\
\hline$\rho_{S} \leq \mathrm{MSE}_{S}$ & High & Only slightly lower than $\mathrm{SNR}_{S}$ \\
\hline$\rho_{W} \leq \mathrm{MSE}_{W}$ & Low & Decreasing function of MSE \\
\hline \multirow{2}{*}{$\mathrm{MSE}_{S}<\mathrm{MSE}_{\text {up }}$} & No MAI & Yes \\
\cline { 2 - 3 } & MAI & Yes \\
\hline \multirow{2}{*}{$\mathrm{MSE}_{W}<\mathrm{MSE}_{\text {up }}$} & No MAI & Yes \\
\cline { 2 - 3 } & MAI & Only if MSE \\
\end{tabular}

line 5-8: Considering up to a few times the root mean square (rms) error value of an estimator, we put an upper bound $\mathrm{MSE}_{\text {up }}$ on the mean square error (MSE) that can be tolerated with a view to symbol detection. In general, this upper bound is largely independent of the SINR.

line 5,7: It is fair to assume that the receiver easily achieves an MSE below $\mathrm{MSE}_{\text {up }}$ when there is no Multiple Access Interference (MAI), i.e., only one terminal is active.

line 6,8: The effect of MAI on the accuracy of conventional estimation structures is different for strong and weak terminals. line 6: Since $\mathrm{SNR}_{S} \gg \mathrm{SNR}_{W}$ and $\mathrm{SNR}_{S}$ is high, the strong terminal NOMA SINR, i.e., $\rho_{S}=\frac{\text { SNR }_{S}}{1+\text { SNR }_{W}}$, is also high. Therefore, it is usually not difficult to obtain estimates $\left(\hat{\tau}_{S}, \hat{F}_{S}, \hat{\theta}_{S}, \hat{A}_{S}\right)$ with an MSE below $\mathrm{MSE}_{\text {up }}$, and it is safe to assume that $\boldsymbol{x}_{S}$ can be detected correctly from $y(t)$ in (15).

line 8: The waveform $f_{S}\left(t ; \boldsymbol{x}_{S}, \hat{\tau}_{S}, \hat{F}_{S}, \hat{\theta}_{S}, \hat{A}_{S}\right)$, is cancelled from (15), and the residual signal $\tilde{y}(t)$ is used for estimating $\left(\tau_{W}, F_{W}, \theta_{W}, A_{W}\right)$ and detecting $\boldsymbol{x}_{W}$.

$$
\tilde{y}(t)=y(t)-f_{S}\left(t ; \boldsymbol{x}_{S}, \hat{\tau}_{S}, \hat{F}_{S}, \hat{\theta}_{S}, \hat{A}_{S}\right)
$$

Due to estimation errors, $\tilde{y}(t)$ still contains MAI. The NOMA SINR of the weak terminal can be expressed as

$$
\rho_{W}=\left(\mathrm{SNR}_{W}\right)\left(1+\mathrm{SNR}_{S} \cdot \mathcal{F}\left(e_{\tau_{S}}, e_{F_{S}}, e_{\theta_{S}}, e_{A_{S}}\right)\right),
$$

where $\mathcal{F}$ is a function that maps the estimation errors $\left(e_{\tau_{S}}=\left|\tau_{S}-\hat{\tau}_{S}\right|, e_{F_{S}}=\left|F_{S}-\hat{F}_{S}\right|, e_{\theta_{S}}=\left|\theta_{S}-\hat{\theta}_{S}\right|\right.$, $\left.e_{A_{S}}=\left|A_{S}-\hat{A}_{S}\right|\right)$ to an average residual interference energy per symbol period, normalized with respect to $A_{S}^{2}$. For small $\left(e_{\tau_{S}}, e_{F_{S}}, e_{\theta_{S}}, e_{A_{S}}\right), \mathcal{F}$ is an increasing function of these estimation errors. Because $\mathrm{SNR}_{S}$ is high, even a slightly larger value of $\left(e_{\tau_{S}}, e_{F_{S}}, e_{\theta_{S}}, e_{A_{S}}\right)$ may result in a significant reduction of $\rho_{W}$. Since $\rho_{W}$ is upper bounded by $\mathrm{SNR}_{W}$, which itself is low, particular attention should be paid to the estimates $\left(\hat{\tau}_{S}, \hat{F}_{S}, \hat{\theta}_{S}, \hat{a}_{S}\right)$ that are used for interference cancellation. An MSE that can be tolerated for detecting $\boldsymbol{x}_{S}$ might be too large for cancelling $f_{S}$. Fortunately, symbol knowledge is available at the time of the cancellation. This allows to reestimate $\left(\tau_{S}, F_{S}, \theta_{S}, a_{S}\right)$ using DA methods. Compared to nonDA methods, DA methods yield a significantly lower MSE.

\section{Time Delay Estimation: Algorithms}

Usually, time delay is estimated before frequency, phase and amplitude. First, the received signal $y(t)$ is applied to a low-pass anti-aliasing filter with bandwidth $\frac{2}{T} \geq B \geq$ $\frac{1}{T}\left(1+\max \left(\alpha_{S}, \alpha_{W}\right)\right)$ and sampled at 4 samples per symbol period. The resulting samples are fed to a discrete-time receive filter, matched to the transmit pulse $h_{S}(t)$ of the strong terminal. Theoretically, $h_{u}(t)(u \in\{S, W\})$ has infinite support but, in practice, it is safe to assume that $h_{u}(t) \approx 0$ for $t \notin\left[-d_{h} T, d_{h} T\right]$ and $d_{h}$ sufficiently large. We obtain

$$
z_{S}(t)=\frac{4}{T} \sum_{m=m_{0}}^{m_{0}+M_{0}-1} h_{S}\left(m \frac{T}{4}-t\right) y\left(m \frac{T}{4}\right),
$$

with $m_{0}>-4 d_{h}, m_{0}+M_{0}-1>4\left(K-1+d_{h}\right)$. Applying the popular non-DA Oerder and Meyr (O\&M) estimator [12] yields

$$
\frac{\hat{\tau}_{S}}{T}=\frac{1}{2 \pi} \arg \left(\frac{1}{M_{0}} \sum_{m=m_{0}}^{m_{0}+M_{0}-1}\left|z_{S}\left(\frac{m}{4} T\right)\right|^{2} e^{j 2 \pi \frac{m}{4}}\right) .
$$

A good rule of thumb is that the maximum MSE that can be tolerated with a view to detecting $\boldsymbol{x}_{u}, u \in\{S, W\}$, needs to be smaller than $10^{-3}$, i.e.,

$$
M S E_{u} \equiv \mathbb{E}\left[\left(\frac{\hat{\tau}_{u}-\tau_{u}}{T}\right)^{2}\right]<10^{-3}, \text { DETECT } \boldsymbol{x}_{u} .
$$

If (21) is fulfilled for $u=S$, and appropriate conditions for frequency offset, phase and amplitude estimates $\hat{F}_{S}, \hat{\theta}_{S}$ and $\hat{a}_{S}$ are met, correct recovery of $\boldsymbol{x}_{S}$ may be assumed. Cancelling the strong terminal waveform from the observation then yields (17). Replacing in (19) and (20) the subscript ' $S$ ' by a subscript 'W', and replacing in (19) the signal $y(t)$ by the residual $\tilde{y}(t)$ an O\&M estimate $\hat{\tau}_{W}$ of $\tau_{W}$ can be obtained. Proper detection of $\boldsymbol{x}_{W}$ requires that (21) holds for $u=W$.

Once $\boldsymbol{x}_{S}$ is known, a maximum-likelihood DA estimate $\hat{\tau}_{S}$ of $\tau_{S}$ can be computed as [13], [14]:

$$
\frac{\hat{\tau}_{S}}{T}=\arg \max _{\tilde{\tau}}\left|\sum_{k=0}^{K-1} z_{S}(k T+\tilde{\tau}) x_{S, k}^{*} e^{-j 2 \pi k \hat{F}_{S} T}\right|,
$$

where $\hat{F}_{S}$ is a previously obtained estimate of $F_{S}$. The implementation complexity of (22) is much higher than that of O\&M (20) [15]. However, (22) can be useful if (20) is not accurate enough to reliably cancel $f_{S}$ from (15).

\section{Time Delay Estimation: Performance Evaluation}

Numerical results with respect to time delay estimation are obtained using Monte-Carlo simulations. First, we numerically evaluate $\mathrm{MSE}_{S}$ and $\mathrm{MSE}_{W}$ for a selection of SNR pairs $\left(\mathrm{SNR}_{S}, \mathrm{SNR}_{W}\right)$ from Table II (i.e., corresponding to a large power imbalance). For $\mathrm{MSE}_{W}$, multiple values of $e_{\tau_{S}}, e_{F_{S}}$, $e_{\theta_{S}}$ and $e_{A_{S}}$ are considered. We observe that:

- In virtually all scenarios of interest, $\mathrm{MSE}_{S}$ is significantly lower than $10^{-3}$.

- Making $\mathrm{MSE}_{W}$ lower than $10^{-3}$ is less trivial and requires large $K$, small $\alpha_{W}$ and small $\left(e_{\tau_{S}}, e_{F_{S}}, e_{\theta_{S}}, e_{A_{S}}\right)$. Next, we assess the sensitivity of $\mathrm{MSE}_{W}$ to $e_{\tau_{S}}{ }^{5}$. In particular, we determine (for each SNR pair) the largest value of $e_{\tau_{S}}$ for

\footnotetext{
${ }^{5}$ For simplicity, the sensitivity to $e_{\tau_{S}}, e_{F_{S}}, e_{\theta_{S}}$ and $e_{a_{S}}$ is assessed separately, i.e., a non-zero estimation error is considered for each parameter successively, while all other parameters are assumed to be perfectly known.
} 
which an $\mathrm{MSE}_{W}$ lower than $10^{-3}$ is achievable. We find a maximum tolerable $e_{\tau_{S}}$ in the order of 0.03 , meaning that an $\mathrm{MSE}_{S}$ of less than ${ }^{6} 10^{-4}$ should be achieved prior to interference cancellation; in other words,

$$
M S E_{S} \equiv \mathbb{E}\left[\left(\frac{\hat{\tau}_{S}-\tau_{S}}{T}\right)^{2}\right]<10^{-4}, \text { CANCEL } \boldsymbol{x}_{S}
$$

The constraint (23) is 10 times stronger than (21). For the considered SNR pairs and the considered values of $K$ and $\alpha_{S}$, an $\mathrm{MSE}_{S}$ smaller than $10^{-4}$ (as opposed to $10^{-3}$ ) is not always achievable with O\&M. Fortunately, (23) must only be fulfilled after detection, namely, at the time of the cancellation. This allows to employ the DA time delay estimator from (22). We have verified that, as opposed to O\&M, the MSE resulting from (22) is usually smaller than $10^{-4}$ under MAI due to NOMA for the considered values of $\mathrm{SNR}_{S}, \mathrm{SNR}_{W}$ and $K$.

\section{CONCLUSIONS}

The application of NOMA techniques for satellite user terminals with a significant imbalance in the link quality has been addressed. The potential gains with respect to more conventional orthogonal allocation schemes have been evaluated in the understanding that some additional complexity at the receivers is required, due to the need for successive interference cancellation schemes.

In the case of the forward link, a super-frame profile is proposed to accommodate PD-NOMA in DVB-S2X. The performance from the application of the proposed super-frame shows gains in the order of $20 \%$ for the overall sum rate, as long as the received SNR gap is above $8 \mathrm{~dB}$ and the weak users have a relevant provision of rate. These results, obtained from the performance of the DVB-S2X MOCDODS with PDNOMA in a linear channel, have been found to degrade in the presence of non-linear power amplifiers as expected, especially for lower SNRs.

As for the return link, the emphasis is given to the heterogeneous coexistence of IoT satellite terminals. Extending the original E-SSA scheme, a new analytical model has been derived assuming the coexistence of two class of terminals with different antenna gains as well as different spreading factors. Numerical results report throughput gains up to $61 \%$ with respect to the orthogonal scheme, when different class of terminals exhibit an imbalance of $18 \mathrm{~dB}$ in transmit EIRP.

Finally, the impact of parameter estimation errors on performance was discussed. A high-level analysis was performed for a demand-assigned pairwise PD-NOMA return link communication system. Specific attention was devoted to time delay estimation. Our numerical results demonstrate that a system that exploits current DVB-RCS waveforms, and pairs strong users (operating at 10 to $15 \mathrm{~dB}$ ) with weak users (operating at 0 to $5 \mathrm{~dB}$ ) is feasible when it comes to synchronization.

Overall, NOMA offers the potential to outperform OMA at the cost of receiver and user pairing (scheduling) complexity. Further system level studies are required to ponder the advantages of NOMA over existing mechanisms.

\footnotetext{
${ }^{6}$ The idea is to consider up to three times the rms value of the estimator.
}

\section{ACKNOWLEDGMENT}

This work has been supported by the European Space Agency funded activity SatNEx IV CoO2-Part 2 WI 2 "NOMA Techniques for Satellite". The views of the authors of this paper do not reflect the views of ESA. This work was partially funded by the Agencia Estatal de Investigacion (Spain) and the European Regional Development Fund (ERDF) through the projects TERESA- TEC2017-90093-C3-1R (AEI/FEDER,UE) and MYRADA (TEC2016-75103-C2-2-R). Also funded by Xunta de Galicia (Secretaria Xeral de Universidades) under a predoctoral scholarship (cofunded by the European Social Fund). This research work was carried out in the frame of Fonds de la Recherche Scientifique - FNRS and Fonds Wetenschappelijk Onderzoek -Vlaanderen FWO EOS Project no 30452698 '(MUSEWINET) MUlti-SErvice WIreless NETwork.

\section{REFERENCES}

[1] A. D. Wyner, "shannon-theoretic approach to a gaussian cellular multiple-access channel," IEEE Transactions on Information Theory.

[2] N. Jindal, S. Vishwanath, and A. Goldsmith, "On the duality of Gaussian multiple-access and broadcast channels," IEEE Transactions on Information Theory, vol. 50, no. 5, pp. 768-783, 2004.

[3] K. Higuchi and A. Benjebbour, "Non-Orthogonal Multiple Access (NOMA) with successive interference cancellation for future radio access," IEICE Transactions on Communications, vol. E98B, no. 3, pp. 403-414, Mar. 2015.

[4] ETSI, "Digital Video Broadcasting (DVB); Second generation framing structure, channel coding and modulation systems for Broadcasting, Interactive Services, News Gathering and other broadband satellite applications; Part II: S2- Extensions (S2-X)," European Standard (EN) EN 302 307-2, 2014

[5] R. De Gaudenzi, O. Del Rio Herrero, G. Gallinaro, S. Cioni, and P.-D. Arapoglou, "Random access schemes for satellite networks, from VSAT to M2M: a survey," International Journal of Satellite Communications and Networking, vol. 36, no. 1, pp. 66-107, 2018. [Online]. Available: https://onlinelibrary.wiley.com/doi/abs/10.1002/sat.1204

[6] O. Del Rio Herrero and R. De Gaudenzi, "High Efficiency Satellite Multiple Access Scheme for Machine-to-Machine Communications," IEEE Transactions on Aerospace and Electronic Systems, vol. 48, no. 4, pp. 2961-2989, October 2012.

[7] ETSI, "Digital Video Broadcasting (DVB); Second Generation DVB Interactive Satellite System (DVB-RCS2); Part 4: Guidelines for Implementation and Use of EN 301 545-2," Technical Report (TR) TR 101 545-4, 2014.

[8] — , "Digital Video Broadcasting (DVB); Second Generation DVB Interactive Satellite System (DVB-RCS2); Part 2: Lower Layers for Satellite Standard," European Standard (EN), Bluebook EN $301545-$ 2, 2019.

[9] D. R. Pauluzzi and N. C. Beaulieu, "A comparison of SNR estimation techniques for the AWGN channel," IEEE Transactions on Communications, vol. 48, no. 10, pp. 1681-1691, 2000.

[10] H. W. Kuhn, "The Hungarian method for the assignment problem," Naval Research Logistics Quarterly, vol. 2, no. 1-2, pp. 83-97, 1955. [Online]. Available: https://onlinelibrary.wiley.com/doi/abs/10.1002/nav.3800020109

[11] N. Alagha, J. Alonso-Zarate, M. Andrenacci, A. Biason, L. Blanco, I. Chesi, D. Tarchi, and M. Zorzi, "Feasibility of Energy Management Techniques for Ultra-low Power M2M SatCom Terminals," in 2018 9th Advanced Satellite Multimedia Systems Conference (ASMS/SPSC), 2018.

[12] M. Oerder and H. Meyr, "Digital filter and square timing recovery," IEEE Transactions on Communications, vol. 36, no. 5, pp. 605-612, May 1988.

[13] M. Casadei, S. Cioni, and G. E. Corazza, "Advanced iterative symbol timing recovery for mobile dvb-rcs," in 2007 IEEE 65th Vehicular Technology Conference - VTC2007-Spring, April 2007, pp. 1425-1429.

[14] C. Herzet, H. Wymeersch, M. Moeneclaey, and L. Vandendorpe, "On maximum-likelihood timing synchronization," IEEE Transactions on Communications, vol. 55, no. 6, pp. 1116-1119, June 2007.

[15] A. Gesell, J. B. Huber, B. Lankl, and G. Sebald, "Dataaided symbol timing estimation for linear modulation," $A E U$ - International Journal of Electronics and Communications, vol. 56, no. 5, pp. 303 - 311, 2002. [Online]. Available: http://www.sciencedirect.com/science/article/pii/S1434841104701028 